\title{
Delimitação automática da Bacia Hidrográfica do Rio Picuí (Estado da Paraíba) através de dados SRTM*
}

\section{José Jerônimo de Souza Nascimentoํㅜ, Thyago de Almeida Silveira², Frederico Campos Pereira ${ }^{2 * *}$}

${ }^{1}$ Docente do Curso de Pós-Graduação em Gestão dos Recursos Ambientais do Semiárido. Instituto Federal de Educação, Ciência e Tecnologia da Paraíba (IFPB). Campus de Picuí. Picuí-PB (CEP 58187-000).

2Professor do Curso de Pós-Graduação em Gestão dos Recursos Ambientais do Semiárido. Instituto Federal de Educação, Ciência e Tecnologia da Paraíba (IFPB). Campus de Picuí. Picuí-PB (CEP 58187-000).*E-mail: fredcampos2000@yahoo.com.br.

Resumo. A bacia hidrográfica é entendida como uma unidade geográfica que compõe um sistema natural, utilizada para planejamento na maior parte do mundo. A qual é de fácil reconhecimento, pois qualquer parte da superfície terrestre está inserida no contexto de uma bacia hidrográfica. Diante do exposto, este trabalho tem como objetivo realizar a delimitação automática da Bacia Hidrográfica do Rio Picuí (BHRP), através de dados SRTM (Shuttle Radar Topography Mission). Para isto, o trabalho foi desenvolvido em três etapas: aquisição dos dados importantes para o trabalho, tratamento destes dados, a partir de técnicas de geoprocessamento. E por fim, análises dos resultados, que possibilitaram compreender a área objeto de estudo. Os resultados apresentaram que a referida bacia hidrográfica possui uma área de drenagem de 756,63 $\mathrm{km}^{2}$ e perímetro igual a $159,67 \mathrm{~km}$, com um padrão de drenagem de estrutura dendrítica. Portanto, conclui-se, que neste trabalho com a metodologia utilizada foi possível gerar informações que podem ser aplicadas em estudos ambientais na mesma ou em outras áreas com características semelhantes.

Palavras-chave: Bacia hidrográfica; Rio Picuí; Geoprocessamento.

Abstract. Automatic delimitation of the Picuí River Hidrographic Basin (State of Paraíba) through the Shuttle Radar Topography Mission (SRTM) data. The watershed is understood as a geographical unit that makes up a natural system, used for planning most of the world. Which is easily recognizable as any part of the earth's surface it fits into the context of a watershed. Given the above, this paper aims at automating the delimitation of the Picuí River Hidrographic Basin (BHRP) through Shuttle Radar Topography Mission (SRTM) data. The study was conducted in three stages: acquisition of important data for work, processing of such data from GIS techniques. Finally, analysis of the results, which made it possible to understand the area object of study. The results showed that the said watershed has a drainage area of $756.63 \mathrm{~km}^{2}$ and perimeter equal to
Recebido:

20/11/2015

Aceito:

27/05/2016

Publicado:

30/06/2016

Acesso Aberto

Artigo completo

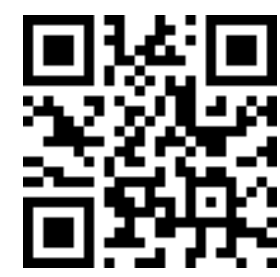

ORCIID

(D) 0000-0002-2351-4165 José Jerônimo de Souza Nascimento

(1) 0000-0003-0874-8999

Thyago de Almeida Silveira

(D) 0000-0003-2715-9508

Frederico Campos

Pereira

*Apresentado no Congresso Brasileiro de Gestão Ambiental e Sustentabilidade - Congestas 2015, João Pessoa-PB, 9 a 11 de dezembro de 2015. 
$159.67 \mathrm{~km}$, with a pattern of dendritic structure of drainage. Therefore, it is concluded that this work with the methodology used was possible to generate information that can be applied in environmental studies in the same or other areas with similar characteristics.

Keywords: Watershed; Picuí River; Geoprocessing.

\section{Introdução}

A bacia hidrográfica serve como unidade de planejamento e gestão ambiental em diferentes países, tendo em vista que consiste em uma unidade espacial de significativo reconhecimento, uma vez que, qualquer parte da superfície terrestre, está inserida no contexto de uma bacia hidrográfica. No Brasil, devido às peculiaridades naturais, a bacia hidrográfica é tida como referência espacial para diferentes estudos físico-territoriais e para implementação de políticas públicas (Rodrigues, 2005). Como por exemplo, a legislação inerente aos recursos hídricos, que define a bacia hidrográfica como unidade territorial para implantação da Política Nacional de Recursos Hídricos (PNRH) e a atuação do Sistema Nacional de Gerenciamento de Recursos Hídricos (SINGREH) (Brasil, 1997).

Assim, conforme Tonello (2005), a bacia hidrográfica é considerada atualmente, a mais adequada unidade de planejamento, pois seus limites são invariáveis, ao considerar o horizonte de planejamento humano, o que possibilita um monitoramento das alterações naturais ou antrópicas. Neste sentido, para a área que compreende o semiárido nordestino, cujas condições edafoclimatológicas apresentam especificidades, tais como evapotranspiração diária elevada, ocorrência de períodos longos de estiagens, solos de pouca profundidade e reduzida capacidade de retenção de água, a compreensão desses espaços geográficos torna-se fundamental.

De forma sucinta, uma bacia hidrográfica pode ser definida como a área limitada por divisores topográficos e composta de uma rede de drenagem formada pelos cursos de água, onde a captação natural da precipitação pluviométrica escoa de forma superficial convergindo para um único ponto de saída, o exutório (Tucci, 1997). Segundo Corseuil e Campos (2007) bacias hidrográficas podem apresentar diferentes dimensões, articuladas entre os divisores de água principais, drenando suas águas em direção a um canal principal, consolidando o sistema de drenagem organizado. Sendo assim, as bacias hidrográficas são consideradas cenários de prováveis e diferentes tipos de degradação, portanto faz-se necessário o monitoramento, com o objetivo de compreender a sua dinâmica diante dos usos de seus recursos naturais, minimizando os efeitos e impactos causados ao ambiente natural (Rocha, 1991).

Nos últimos anos o uso do geoprocessamento tem apresentado significativa relevância, tendo em vista a sua utilização em diferentes setores de trabalho, tais como análises ambientais, comunicação, transporte, energia, dentre outros, auxiliando no planejamento territorial urbano e rural. Este cenário pode ser atribuído ao crescimento das tecnologias da informática que possibilita a qualquer indivíduo, com certo conhecimento, realizar de forma rápida e com precisão, a resolução de determinado problema. No que diz respeito ao conceito de geoprocessamento, Rosa (2005) define como o conjunto de tecnologias utilizadas para coletar, processar, analisar e disponibilizar informações georreferenciadas. Já Câmara et al. (1999) afirmam que este termo consiste em uma área do conhecimento que utiliza métodos matemáticos e computacionais para o tratamento de informações geográfica.

Portanto, considerando a importância do gerenciamento dos recursos naturais, entre eles os recursos hídricos, o uso de técnicas de geoprocessamento permite a obtenção qualitativa e quantitativa de dados geográficos que auxiliam na gestão destes 
recursos. Para tanto, o uso de imagens orbitais são fontes de dados relevantes para a aplicação nas análises em bacias hidrográficas. Entre estas imagens podem ser utilizadas às oriundas da Shuttle Radar Topography Mission (SRTM). No entanto, Souza et al. (2013) afirmam que apesar das informações obtidas a partir dos dados SRTM apresentarem relevante potencial de utilização, para estudos físico-ambientais em bacias hidrográficas, tais dados requer um processamento específico para este fim. Assim, este trabalho tem o objetivo de realizar a partir de processos automáticos a delimitação da Bacia Hidrográfica do Rio Picuí (BHRP), através de dados SRTM, tendo em vista a sua importância para a Microrregião do Seridó Oriental Paraibano.

\section{Metodologia}

\section{Área de estudo}

A área objeto de estudo deste trabalho corresponde a Bacia Hidrográfica do Rio Picuí (BHRP), localizada no Semiárido Paraibano, especificamente na Mesorregião Geográfica da Borborema no Estado da Paraíba entre as coordenadas geográficas de latitudes $6^{\circ} 12^{\prime} 00^{\prime}$ S e $6^{\circ} 46^{\prime} 00$ ” S e Longitudes $36^{\circ} 27^{\prime} 00^{\prime}$ ' W e $36^{\circ} 10^{\prime} 00^{\prime} \mathrm{W}$. A referida bacia hidrográfica possui uma área de drenagem corres

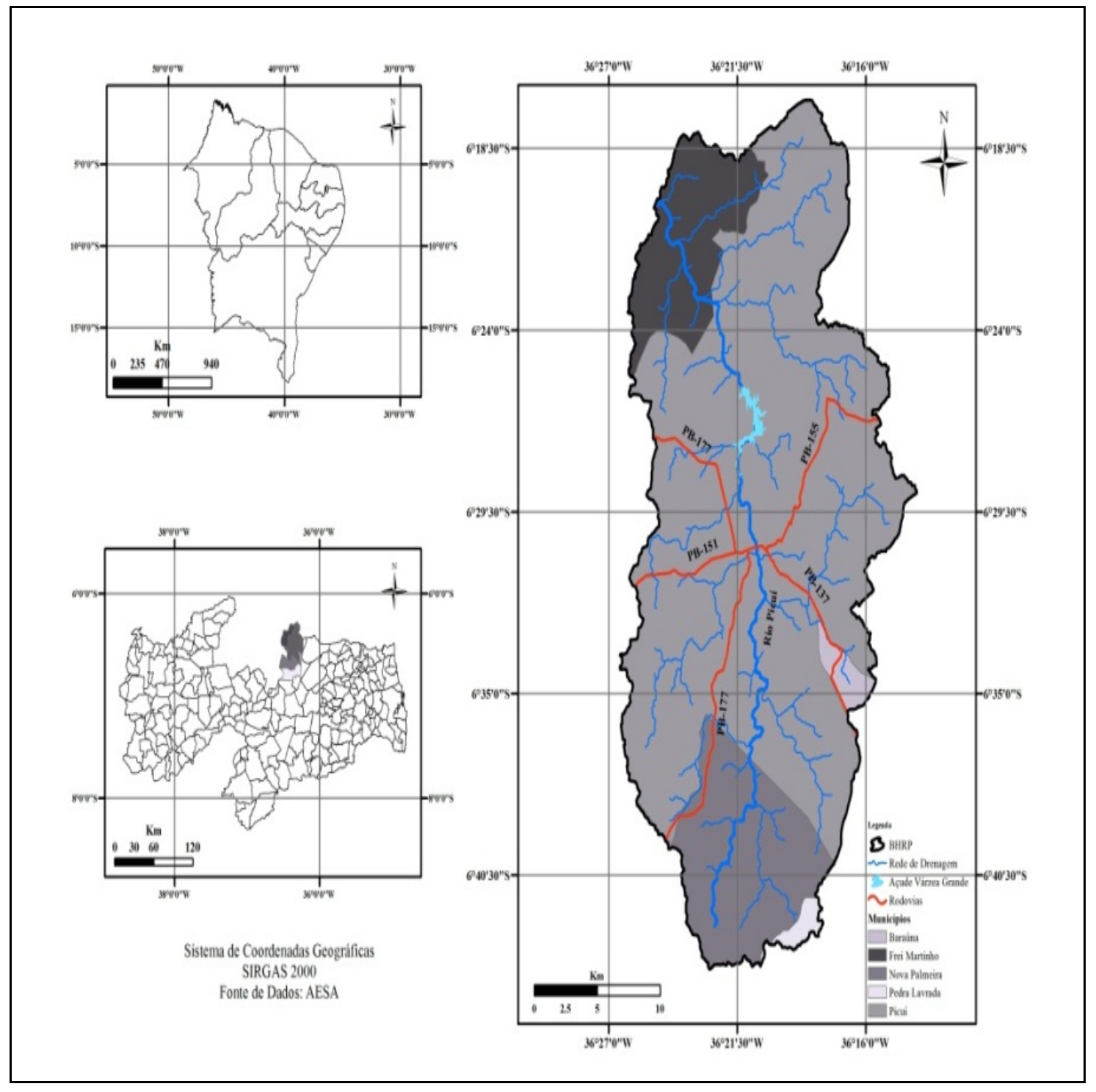

Figura 1. Mapa de localização da Bacia Hidrográfica do Rio Picuí. 
pondente a 756,63 km², compreendendo partes dos Municípios de Baraúna, Frei Martinho, Nova Palmeira, Pedra Lavrada e Picuí (Figura 1).

\section{Procedimentos metodológicos}

De forma sucinta, o trabalho foi desenvolvido em três etapas: aquisição dos dados, tratamento dos dados e analise dos dados. Na primeira etapa os dados foram obtidos junto ao Instituto de Pesquisas Espaciais (INPE), do Projeto TOPODATA. No formato GEOTIFF (16 bits), resolução espacial de $30 \mathrm{~m}$, Sistema de Coordenadas Geográficas e Datum WGS-84. Os quais são derivados dos dados SRTM, desenvolvidos pela National Aeronautics and Space Administration (NASA) e National Geospatial-Intelligence Agency
(NGA). Na segunda etapa, o tratamento dos dados foi realizado a partir do uso de técnicas de geoprocessamento. Para tanto, foi utilizado o software de SIG, ArcGIS 10.1. Assim, no processamento dos dados, adotou-se o Sistema de Coordenadas Geográficas e o Datum SIRGAS 2000 para a zona 24 Sul. Desta forma, a caracterização física da Bacia Hidrográfica do Rio Picuí foi realizada a partir da extração do Modelo Digital de Elevação (MDE) da imagem, conforme as seguintes etapas:

1 - preenchimento de depressões;

2 - direção de fluxo;

3 - fluxo acumulado;

4 - delimitação de bacias como demonstra a (Figura 2).

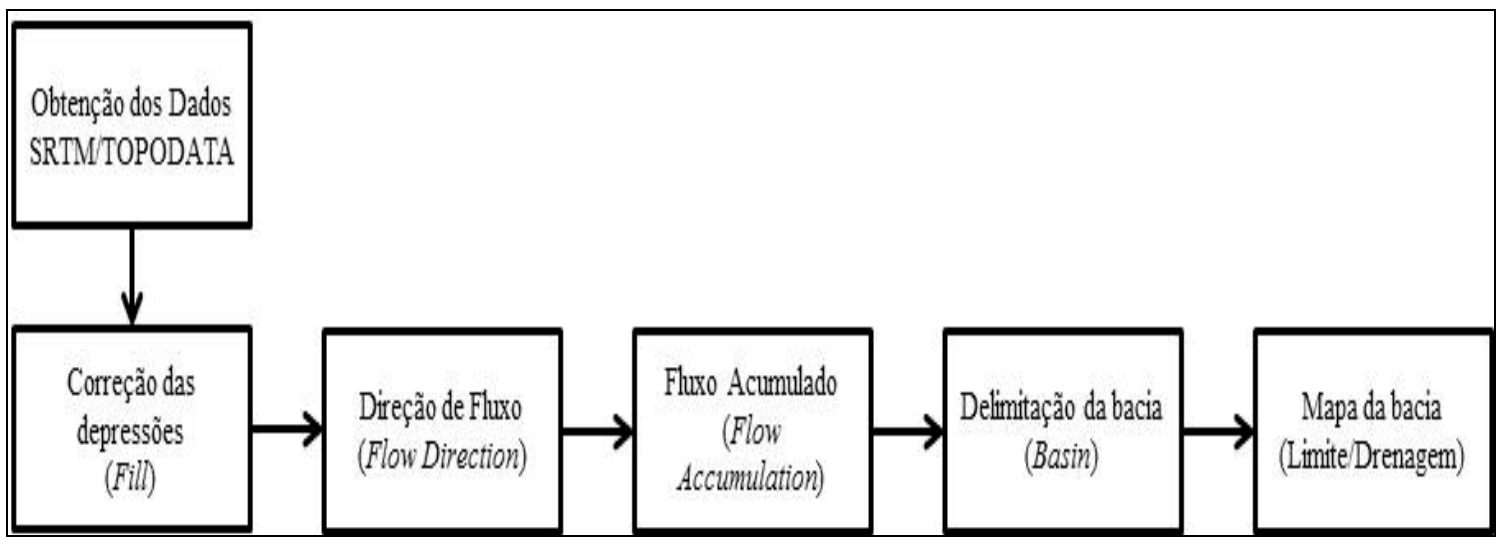

Figura 2. Fluxograma das etapas para delimitação da BHRP a partir de dados SRTM.

E por fim, na terceira etapa, foram feitas as análises dos resultados e discussões, possibilitando a compreensão da área objeto de estudo.

\section{Resultados e discussão}

Como resultado deste trabalho, são expostos mapas elaborados a partir dos processos realizados na extração do Modelo Digital de Elevação (MDE). Logo, devido à forma de imageamento do radar, os dados do SRTM podem apresentar depressões oriundas das feições naturais ou artificiais do relevo. Como por exemplo, áreas que apresentam inclinações da superfície maiores que 20 graus, presença de corpos hídricos e vegetação arbórea densa. Com a utilização de SIG, essas depressões foram corrigidas utilizando as altitudes dos pixels vizinhos da imagem para preencher essas depressões no MDE (Figura 3). O preenchimento das depressões, foi possível definir a direção de fluxo do escoamento da água, a partir da maior declividade do terreno, utilizando-se da função. De posse da direção de fluxo obteve-se o valor do fluxo acumulado através da função, o qual diz respeito ao valor do acúmulo de água dentro de cada pixel correspondente a rede 
hidrográfica da área da BHRP (Figuras 4 e 5).

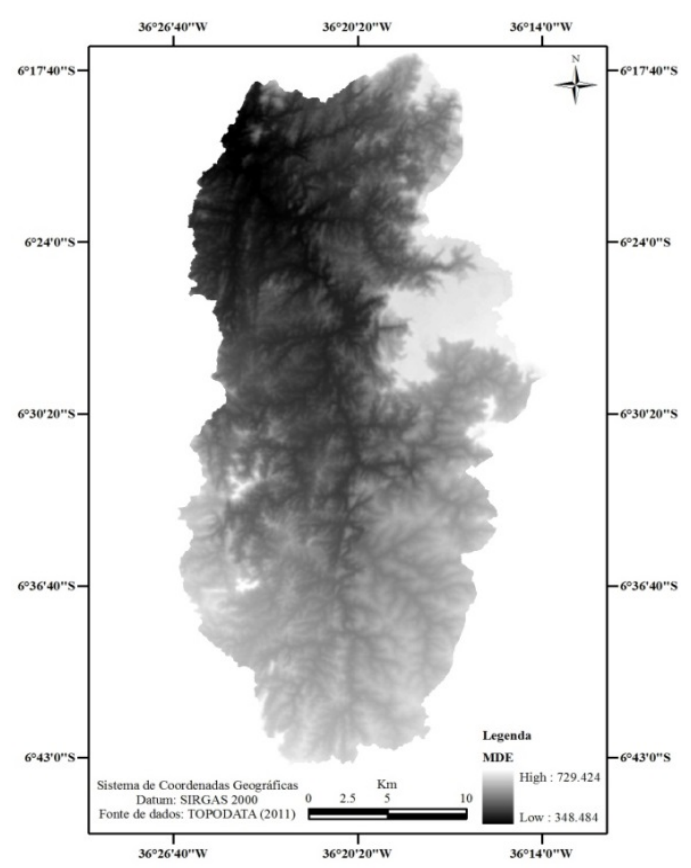

Figura 3. Mapa de preenchimento das depressões (Fill) do MDE da BHRP.

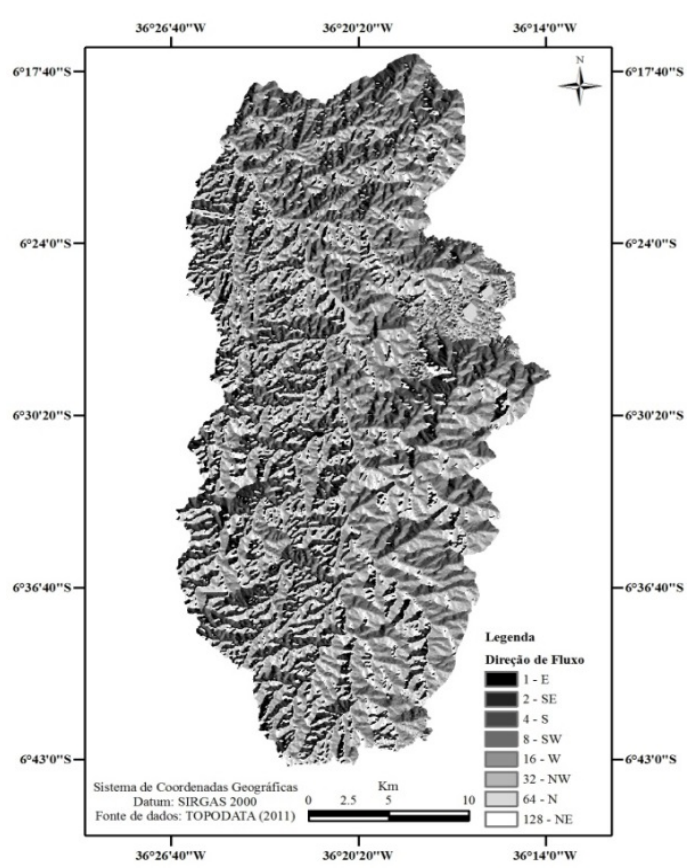

Figura 4. Mapa de Direção de fluxo da BRHP.

Por fim, a Figura 6 apresenta a delimitação e a rede de drenagem da Bacia Hidrográfica do Rio Picuí extraída a partir dos procedimentos realizados. A referida bacia hidrográfica dispõe de uma área de drenagem de $756,63 \mathrm{~km}^{2}$ e perímetro de $159,61 \mathrm{~km}$. Portanto, diante do processamento realizado pode-se afirmar que a BHRP apresenta um padrão de drenagem de estrutura dendrítica. Correlacionando-se com os aspectos geomorfológicos, condições climáticas e de vegetação da região.

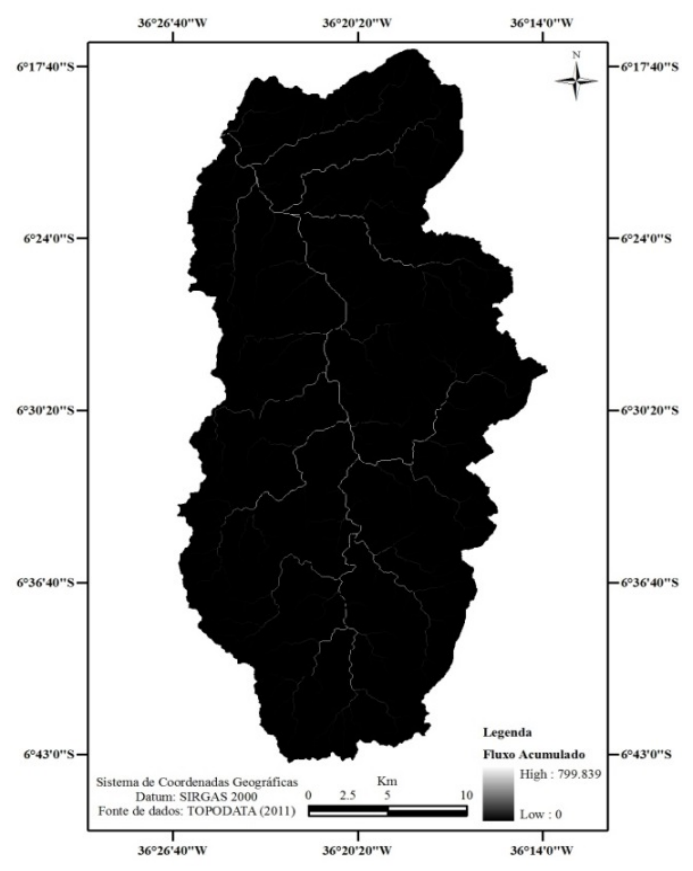

Figura 5. Mapa de Fluxo acumulado da BRHP.

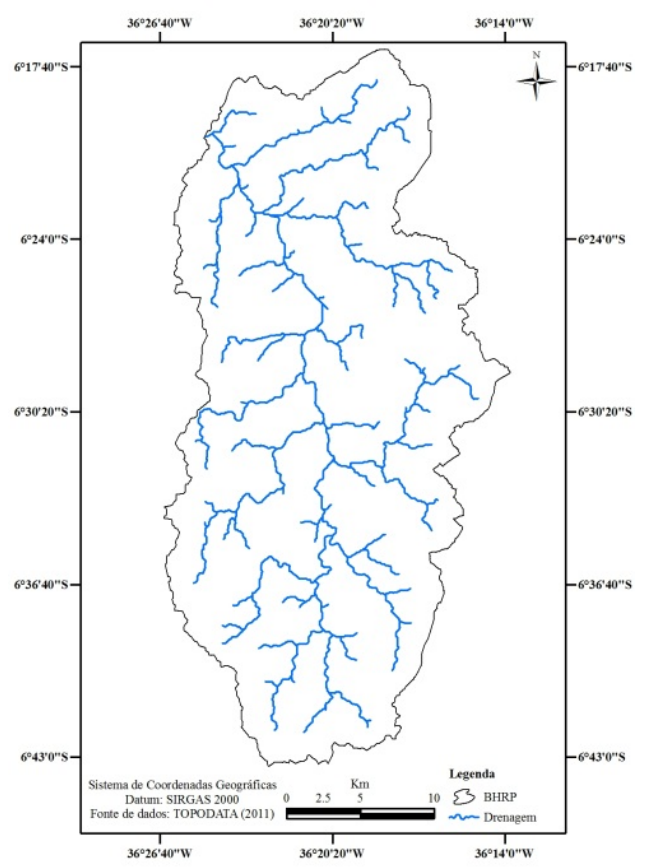

Figura 6. Mapa de rede de drenagem da BRHP. 


\section{Conclusões}

Os resultados obtidos a partir dos procedimentos metodológicos aplicados neste trabalho possibilitaram expor uma compreensão inicial das características físicas, no que diz respeito ao limite e ao padrão de drenagem da Bacia Hidrográfica do Rio Picuí. O uso de técnicas de geoprocessamento mostrou-se satisfatório, uma vez que, permitiram gerar informações importantes sobre a referida bacia hidrográfica, atendendo ao que se propôs nos objetivos do trabalho.

Tais informações podem servir de embasamento para possíveis tomadas de decisões no âmbito da bacia hidrográfica. Mas, com uma ressalva, a de que para uma aplicação mais específica, faz-se necessário a realização de estudos mais detalhados. Ao avaliar os resultados sobre a Bacia Hidrográfica do Rio Picuí, considera-se a possibilidade de diferentes estudos ambientais sobre a área, tendo em vista sua importância para os municípios inseridos, em especial, o de Picuí-PB.

Portanto, este trabalho torna-se relevante à medida que apresenta uma possibilidade, diante de inúmeras, de desenvolver estudos na área ambiental no âmbito de uma bacia hidrográfica, a partir do mesmo procedimento metodológico.

\section{Declaração de conflito de interesses}

Os autores declaram não haver conflito de interesses.

\section{Referências}

Brasil. Leis, Decretos, etc. Lei $\mathbf{n}^{\mathbf{0}} \mathbf{9 . 4 3 3}$, de 8 de janeiro de 1997. Institui a Política Nacional e Sistema Nacional de Gerenciamento de Recursos Hídricos. Disponível em: $<$ http://www.planalto.gov.br/ccivil_03/leis/L943 3.htm>. Acesso em: 20 out. 2015.
Câmara， G.; Barbosa, C. C. F.; Davis, C.; Fonseca, F. (Orgs.). Conceitos básicos em geoprocessamento: teoria e aplicações. São José dos Campos: INPE, 1999.

Corseuil, C. W.; Campos, S. Geoprocessamento aplicado na determinação das classes de declive e de uso das terras da Microbacia do Arroio Ajuricaba - Marechal Cândido Rondon - PR. Energia na Agricultura, v. 22, n. 1, p. 33-41, 2007. Disponível em: $<$ http://revistas.fca.unesp.br/html/CD_REVIST A_ENERGIA_vol9/vol22n12007/artigos/Claudi a Weber Corseuil.pdf $>$. Acesso em: 20 out. 2015.

Rocha, J. S. M. Manual de manejo integrado de bacias hidrográficas. Santa Maria: Edições UFSM, 1991.

Rodrigues, C.; Adami, S. Técnicas fundamentais para o estudo de bacias hidrográficas. In: Venturi, L. A. B. Praticando a Geografia: técnicas de campo e laboratório em Geografia e análise ambiental. São Paulo: Oficina de Textos, 2005. p. 147-166.

Rosa, R. Geotecnologias na geografia aplicada. Revista do Departamento de Geografia, n. 16, p. 81-90, $2005 . \quad$ Disponível em: $<$ http://www.geografia.fflch.usp.br/publicacoes/ RDG/RDG_16/Roberto_Rosa.pdf>. Acesso em: 20 out. 2015.

Souza, C. H. W.; Bazílio, S.; Wrublack, S. C.; Mercante, E.; Boas, M. A. V. Delimitação automática da Microbacia Hidrográfica do Rio das Lontras, através de dados SRTM. Anais XVI Simpósio Brasileiro de Sensoriamento Remoto, Foz do Iguaçu, p. 5515-5522, 2013. Disponível em: <http://www.dsr.inpe.br/ sbsr2013/files/p0277.pdf>. Acesso em: 20 out. 2015.

Tonello, K. C. Análise hidroambiental da Bacia Hidrográfica da Cachoeira das Pombas, Guanhões, MG. Viçosa: Universidade Federal de Viçosa, 2005. (Dissertação de Mestrado em Ciência Florestal). Tucci, C. E. M. Hidrologia: ciência e aplicação. 2. ed. Porto Alegre: ABRH/ Editora da UFRGS, 1997. 